\title{
Infection by Mycobacterium leprae of household contacts of lepromatous leprosy patients from a post-elimination leprosy region of Colombia
}

\author{
Nora M Cardona-Castro/ ${ }^{+}$, Sandra Restrepo-Jaramillo, Myriam Gil de la 0 ssa*, \\ PatrickJ Brennan**
}

Laboratorio de Microbiología, Instituto Colombiano de Medicina Tropical-Instituto de Ciencias de la Salud, CES, Cra. 43 A No. 52 Sur 99 Sabaneta, AA 52162, Medellín, Colombia *Empresa Social Del Estado, Metrosalud, Medellín, Colombia **Department of Microbiology, Immunology and Pathology, Colorado State University, Fort Collins, Colorado, US

The Leprosy Control Program of Antioquia, (post-elimination leprosy state of Colombia), had registered by 1999, 56 lepromatous leprosy patients and their household contacts (HHC). Our interest was to detect Mycobacterium leprae infection in these HHC. Clinical examination, acid-fast bacillary staining (AFB) in nasal secretions, and slit skin samples, IgM anti-PGL-I in serum and Lepromine A (Mitsuda) reactivity were tested. Two hundred forty eight HHC were studied, 49\% were male. After clinical examination, two HHC were diagnosed as multi bacillary patients; $13 \%$ showed positive IgM anti-PGL-I titers; Mitsuda reaction ( $\geq 4 \mathrm{~mm})$ was positive in 59\%; AFB was negative in all samples, except in the two new patients.

HHC were classified according to test results. Group 1: two new multi bacillary patients. Group 2: 15 HHC seropositive, Mitsuda-negative. Group 3: 13 HHC seropositive, Mitsuda-positive. Group 4: 130 HHC seronegative, Mitsuda-positive. Group 5: 88 HHC seronegative, Mitsuda-negative. These results are an indication that the transmission of the infection is still happening in a region considered in the post elimination phase. The two new patients represent an infection source for others contacts, and groups 2 and 3 are infected HHC that could develop the disease in future. Follow up of high risk population is necessary to achieve real elimination of leprosy.

Key words: post elimination leprosy region - household contact infection - Mycobacterium leprae - Colombia

The total number of leprosy cases around the world is decreasing; at the beginning of 2003 the total of cases was 534,000 (WHO 2004). The principal factor contributing to this success worldwide has been the implementation of the standardized multiple drug therapy (MDT) regimen against the causative agent Mycobacterium leprae. Leprosy elimination campaigns for case detection in communities, training of physicians and leprosy field workers, and promotion of public awareness are additional key elements in this infectious disease control approach. Actually, WHO has a commitment to "the final push to reach elimination by 2005", a target originally set for 2000 (WHO 2003). However, the opinions of experts in clinical and research field range from optimistic to pessimistic concerning the reach of this target (Naafs 2000, Visschedijk et al. 2000, Lockwood 2002).

The apprehension arises from the incomprehensible incidence rate that is still high in six countries (4.19 per $10,000)$, which represents $88 \%$ of the world's new cases detected in 2002, and the insufficiency of consistent in-

Financial support: Pan-American Health Organization, Empresa Social del Estado Metrosalud, Dirección Seccional de Salud de Antioquia, The Nacional Institutes of Health, Instituto Colombiano de Medicina Tropical

+Corresponding author. E-mail: ncardona@ ces.edu.co

Received 23 May 2005

Accepted 21 September 2005 formation on the core elements of this infectious disease, namely, what is the source, the reservoir and the mode of transmission, and the host factors related to immunity or disease (Reich 1987, Naafs et al. 2001). Additionally, while prevalence has been reduced, since MDT implementation, there has not been a compelling difference in the number of newly detected cases in the same period and population (incidence rates) before and after introduction of MDT (Feenstra 2003).

The prevalence of leprosy in Colombia has decreased over the last 15 years. While in 1985 , leprosy prevalence was $5,5 / 10,000$, it is now reduced to $0,5 / 10,000$ (www.minsalud.gov.co). The introduction of MDT in Colombia in 1985 has contributed to the decrease in disease burden. According to WHO, leprosy in Colombia is not a public health problem, and control of leprosy is in the "post-elimination phase" (Pinto 2000).

Nevertheless, the reduction in prevalence is not a real reflection of the problem with respect to population distribution. For instance, Colombia has some regions where the actual prevalence is 1 to $3 / 10,000$ and other regions where prevalence is 0,2 to $0,5 / 10,000$. Moreover, the "incidence" (new cases) of leprosy in Colombia in 2002 was $0,7 / 100,000$ habitants, and some specific states reported an incidence of 4,1/100,000 (Cesar state); Magdalena state reported a new case detection rate of 3,46/100,000 habitants (www.minsalud.gov.co), reflecting the fact that because of the long period of incubation of leprosy ( 2 to 20 years), infected contacts may develop disease long after the index case has been treated and cured (Ottenhoff 1994). 
The compulsory plan for health attention in Colombia includes the clinical examination of household contacts (HHC) at the moment of the diagnosis of the patient (index case). While this is a sound concept for surveillance measures, strategic, and logistic problems in maintaining this program exist (Kalk \& Fleisher 2004).

Leprosy is a slow onset disease, and the symptoms are difficult to perceive at the early stages of infection. Self healing does occur in a large proportion of those infected. Those that do have symptoms may not ascribe them to leprosy, thus clinical diagnosis is often delayed which makes it difficult to identify the immediate causes and factors that contribute to the onset of this disease. Clinical diagnosis of leprosy is possible only when the patient is symptomatic, exhibiting lesions and damage to target tissues (Ottenhoff 1994).

A significant proportion of new cases are among children (WHO 2004) despite good MDT coverage, which reflects the inadequacy of MDT to curb recent transmission. It is also not known whether MDT has had an impact on reducing the number of MB cases (WHO 2003); an indicator of this premise is the high number of cases with leprosy related disabilities (Grade 2). Drug resistance to MDT is also a new problem, as indicated by the number of relapses among treated cases (WHO 2004).

Given these indicators, and the reduction in the emphasis on specialized leprosy programs which are being absorbed into the mainstream health care in most countries, it is feared that trained leprologists and leprosy workers will become rare and leprosy cases may be missed because of inadequate diagnosis (Naafs 2000, Kalk \& Fleisher 2004). Failure to diagnose is not a problem confined to countries where leprosy is still recognized as a public health problem. In countries where prevalence has fallen to a rate below 1 in 10,000 the problem may actually be increasing. The less common a disease the less likely a doctor is to consider the diagnosis.

HHCs of leprosy patients are the risk population that has to be monitored for early detection of the disease. Several approaches had been used to diagnose infection and the early stages of disease, which are considered to be important towards future efforts at leprosy elimination (Chanteau et al. 1993, Van Beers et al. 1994, Wu et al. 1999, Torres et al. 2003).

The approach for the detection of infection in HHC in a region considered in the post-elimination phase, used in this study, was the evaluation of this population using several tests at the same time, namely: clinical examination to detect suspected lesions or signs of leprosy, detection of IgM anti PGL-I antibodies as a measure of previous exposure to M. leprae, Ziehl Neelsen staining to detect acid-fast bacilli in nasal secretions and in slit skin samples. Also, the evaluation of the cellular immune response (Mitsuda reaction), was performed as a predictor measure of the immune susceptibility to develop different clinical outcomes of leprosy in infected HHC.

\section{MATERIALS AND METHODS}

A cross sectional survey was performed in $\mathrm{HHC}$ of leprosy patients registered in the leprosy control program of Antioquia state of Colombia.
Study leprosy population - HHC: 248 HHC of 56 lepromatous leprosy (LL) patients were recruited. These were defined as volunteers over four years of age, living in the same home as the leprosy patient for more than two years. Leprosy patients: individuals with diagnosis of LL according to Ridley and Jopling (1966), under or after treatment.

These populations come from several cities and towns located in Antioquia state, a post elimination leprosy region of Colombia. An informed consent form was signed by each participant and tutors of children under 18 , before inclusion in the study.

Serology - IgM antibodies to PGL I were tested in blood samples using an ELISA test. Serum was obtained by spinning the blood samples at $3500 \mathrm{rpm} 10 \mathrm{~min}$ and diluted at 1:40. The ELISA test was based on a synthetic conjugate containing the carbohydrate epitope of PGL I. The test employed anti-human-IgM alkaline phosphatase and a fluorescent substrate, 4-metilumbelipheril phosphate, which, when hydrolyzed, emits fluorescence which is detected by an illuminometer (SUMA model 421 B equipment UMELISA HANSEN UM 2008 Tecnosuma, Havana, Cuba) (Cho et al. 1983, Meeker et al. 1986, Mora et al. 1992). The cut-off for interpretation positive and negative IgM anti-PGL-I titers was calculated using serum samples from 75 individuals of the general population of Medellín city, with similar socioeconomic conditions of the studied population. A positive result was interpreted according to the calculated cut-off value 0.394 (mean plus 3 standard deviations).

Lepromin A skin test - This intradermal test made use of Lepromin A prepared from M. leprae-infected armadillo tissue (provided by Schieffelin Leprosy Research and Training Center, Karigiri, Vellore, India). Lepromin A (0.1 $\mathrm{ml}$ of 37 millions of bacilli/ml) was injected intradermally into the lateral side of the right forearm, and the induration was measured at 21-28 days after application, representing the Mitsuda reaction. Reactions of $\geq 4 \mathrm{~mm}$ were considered positive. A positive Mitsuda reaction indicates that if a person is infected, he/she will most likely develop tuberculoid leprosy, whereas if the Mitsuda test is negative and if the person gets infected, he/she may develop lepromatous leprosy disease (Sengupta 2000).

Acid-fast bacillar staining - The Ziehl Neelsen stain was carried out on nasal secretions and lymph samples according to procedures described (Isenberg 1992).

Clinical examination - Each of the HHC was examined clinically, searching for skin and neurological signs of leprosy. BCG scar was documented. A clinical record was filled including the following data: the leprosy case (index case), date of the leprosy diagnosis, gender, age, bacillary index when diagnosis was done, current bacillary index, current state of treatment, kind of family relationship, socio-economic status; with respect to the HHC: age, gender, time of contact with the index case, socioeconomic status, IgM anti-PGL I titers, results of AFB stain, and Mitsuda reaction.

Data analysis - Statistical analyses were performed using the EPI info 6.04 statistical program. Comparison 
between qualitative variables was done by Chi-square test; quantitative variables were compared by t-Student test. The significant statistic level was $\mathrm{p}=0.05$.

\section{RESULTS}

Leprosy patients - Fifty-six LL patients, 13 women and 43 men, were registered with their HHC in the leprosy program control of Antioquia state of Colombia. The mean age of the 56 leprosy patients was 54.3 years, within a range of 26 to 84 years of age $(\mathrm{SD} \pm 15.2)$. Twenty-seven patients were receiving leprosy treatment (46.2\%) and 29 $(53.8 \%)$ patients were under surveillance. A ratio of $1: 3$ female to male was present in this group of patients (13:43). At the moment of the study, 48 patients showed a bacillary index $(\mathrm{BI})=0$, four patients had $\mathrm{BI}=1$, two patients had $\mathrm{BI}=2.4$, and two $\mathrm{aI}=3$.

According to gender, the mean age of females was 55.2 years, a minimum age of 30 years and a maximum age of 74 years $(\mathrm{SD} \pm 14.3)$. Twelve women had $\mathrm{BI}=0$, and one had $\mathrm{BI}=3$, six of them under treatment. The mean age of the male group was 54 years, with a maximum of 84 years and a minimum of 26 years $(\mathrm{SD} \pm 15.7)$. Thirty-six male patients had $\mathrm{BI}=0$, four $\mathrm{BI}=1$, two $\mathrm{BI}=2.4$, and one $\mathrm{BI}=$ 3 . Twenty three male patients were under treatment.

$H H C$ - The mean age of the HHC was 38.5 years, ranging from 4 to 92 years, $(\mathrm{SD} \pm 24.8) ; 49 \%$ were male.

According to gender, the mean age in the female group was 30.5 years with a range of 4 to 72 years $(S D \pm 18)$. The mean age in the male group was 49.5 with a maximum of 92 years and a minimum age of 5 years $(S D \pm 26.5)$.

The socio-economic status of the studied population corresponded to grades 1,2 , and 3 on a scale of 1 to 6 (6 being the highest socio-economic status, according to Colombian government classification). The familiar relationship of $\mathrm{HHC}$ with the leprosy patient was: $5.2 \%$ spouses, $22.5 \%$ sons/daughters, $5.8 \%$ parents, and $66.5 \%$ others (sister/brother, son/daughter-in law, uncle/aunt, niece/nephew, cousin, and grandchild).

Mitsuda reaction - The Mitsuda reaction was positive ( $\geq 4 \mathrm{~mm}$ ) in $59 \%$. The mean age of the Mitsuda reaction-negative individuals was higher (54.3 years of age) than the mean age of individuals with Mitsuda reactionpositive (33 years); this difference was significant, $\mathrm{p}=$ 0,0001 .

Comparison of the time of contact of the $\mathrm{HHC}$ with the patient was analyzed with respect to Mitsuda reaction. It was found that the mean time of contact of the Mitsuda reaction-positive $\mathrm{HHC}(15,05$ years) was not different with respect to the mean time of contact of $\mathrm{HHC}$ with Mitsuda reaction-negative $(13,12$ years); this difference was not significant, $\mathrm{p}=0,22$.

The mean of IgM anti PGL-I titers $(0,26 \mathrm{SD} \pm 0,22)$ in Mitsuda reaction-negative individuals, was not different with respect to the mean antibody titers $(0,22 \mathrm{SD} \pm 0,30)$ found in HHC Mitsuda reaction positive, $\mathrm{p}=0,07$.

ELISA test for IgM anti PGL-I - IgM titers against PGL-I were positive in $13 \%$ of the studied population. The IgM anti-PGL-I titers considered positive and negative were taken into account to compare the different mean age. The mean age was higher $(54,1$ years old) for the individuals with positive titers than the mean age of individuals with negative titers (37,3 years old); this difference was significant, $\mathrm{p}=0.0000001$.

The mean of contact time with the leprosy patient found in HHC IgM anti PGL-I positive was 13,7 years. This mean of contact time did not show difference with respect to the mean of contact time in HHC IgM anti PGL-I negative $(13,7$ years $),(\mathrm{p}=0,83)$.

BCG scar - BCG scar was detected in $88 \%$ of the volunteers. It was found that its presence was not related to the IgM anti-PGL-I positive titers, or Mitsuda reaction positivity: $p>0,05$ respectively.

Clinical examination - Two HHC showed clinical signs of leprosy in skin (hypochromic lesions); AFB stain was positive (bacillary index, 2.4 for both new patients). Both individuals had positive titers for IgM anti-PGL-I, Mitsuda reaction-positive, and presence of BCG scars. For purposes of treatment and due to the $\mathrm{BI}>0$, they were classified as MB leprosy patients and were included in the leprosy program for therapy. The new MB patients are relatives (brother and sister), 21 and 18 years old respectively. They were contacts of their father; a LL patient that was under treatment during one year, at the moment of the survey. Both were evaluated by the leprosy program when the index case was diagnosed; at this time they were not diagnosed as leprosy patients.

Acid-fast stain - The acid-fast stains were negative in all samples tested, except in the two new patients.

Classification of $\mathrm{HHC}$ - $\mathrm{HHC}$ were classified into five groups according to the results of the tests (Table). Group 1 consisted of two HHC diagnosed as MB patients. Group 2 consisted of $15 \mathrm{HHC}$ with IgM anti-PGL-I-positive titers and Mitsuda reaction negative. Group 3 consisted of 13 HHC, all IgM anti-PGL-I-positive titers and Mitsuda reaction positive. Group 4 was composed of $130 \mathrm{HHC}$, seronegative and Mitsuda reaction positive. Group 5 consisted of $88 \mathrm{HHC}$ negative for IgM anti-PGL-I titers and also Mitsuda reaction negative.

TABLE

Classification of household contacts of lepromatous leprosy patients into five groups based on acid-fast stain, Mitsuda reaction, and IgM anti-PGL-I

\begin{tabular}{lccc}
\hline $\begin{array}{l}\text { Group } \\
(\mathrm{n})\end{array}$ & $\begin{array}{c}\text { Mitsuda } \\
\text { positive }\end{array}$ & $\begin{array}{c}\text { Acid fast bacillar } \\
\text { stainpositive }\end{array}$ & $\begin{array}{c}\text { IgManti-PGL-I } \\
\text { positive }\end{array}$ \\
\hline $1(2)$ & 2 & 2 & 2 \\
$2(15)$ & 0 & 0 & 15 \\
$3(13)$ & 13 & 0 & 13 \\
$4(130)$ & 130 & 0 & 0 \\
$5(88)$ & 0 & 0 & 0 \\
\hline Total & $145 / 248$ & $2 / 248$ & $30 / 248$ \\
\hline
\end{tabular}




\section{DISCUSSION}

$\mathrm{HHC}$ of MB patients have long been regarded as those with the highest risk of developing clinical leprosy. An active follow up of $\mathrm{HHC}$ of leprosy patients, in regions where leprosy prevalence has dropped to $<1 / 10.000$, as is the case of Antioquia state, is important in order to maintain leprosy within the group of diseases due for elimination as a public health problem, however this follow up is not included in the Colombian leprosy control program.

It is important to note that in this survey, two new MB patients among $\mathrm{HHC}$ evaluated $(0.8 \%)$ were detected at the initial state of the disease, without important neuronal and/or physical irreversible damage. These two HHC were evaluated one year ago but they were not diagnosed as leprosy patients, suggesting that the skin lesions that the two new patients show were not detected or not attributed to leprosy at this time. These results are an indication that the follow up of the HHC is useful for early detection of leprosy. In addition, results confirm that transmission of the infection is still happening in a region considered in the post elimination phase. These two new MB patients represented an infection source for others contacts.

The application of simple laboratory-based tests described here now allows a new at-risk classification of such contacts, which is supported by the knowledge of the immunology of leprosy in patients (Ottenhoff 1994).

According to leprosy immunology, Group 2 was classified as at risk to develop LL, since the $15 \mathrm{HHC}$ were infected (anti-PGL-I IgM positive titers) and Mitsuda reaction negative. Group 3 of $13 \mathrm{HHC}$ were also infected as shown by the anti-PGL-I positive titers. However, they were Mitsuda-positive, meaning that if they develop leprosy, they will have the tuberculoid form (Ottenhoff 1994, Sengupta 2000).

Groups 2 and 3 are infected HHC that according to immune condition, could develop the disease in future; they are the most important population detected by this survey that have to be followed for documentation of changes and possible early diagnostic of leprosy.

Groups 4, $130 \mathrm{HHC}$ were seronegative and positive for the Mitsuda reaction. Thus, this group should have a good immune response in case of future infection.

Group 5 of $88 \mathrm{HHC}$ were negative for both, anti-PGL-I antibodies and Mitsuda reaction. They are considered non-infected, however, if they become infected, it appears likely that this population will be at more risk to develop LL, according to their negative Mitsuda reaction.

As groups 2 and 3, HHC classified in group 4 and 5 belong to a high risk population to develop leprosy due to immunological and/or epidemiological antecedents of leprosy contact. This population would benefit from periodic follow up; however, as mentioned before, leprosy control program does not include this measure as a leprosy control activity.

Current results raise some questions about the necessity of include control measures of leprosy in regions considered in the post elimination phase. The follow up will be more effective if it is included for high-risk populations using not only clinical examination but also labora- tory diagnostic tools. These tools should include direct tests to determine the presence of $M$. leprae (acid-fast stain) in nasal secretions and slit skin samples and, also, detection of immune responses through Lepromin A testing and specific antibody tests. All of the mentioned tests are useful as indicators of infection and immune response in HHC (Ottenhoff 1994, Wu et al. 1999, Sengupta 2000).

The WHO (1998) has indicated that a priority in leprosy research is to develop technology capable of diagnosing leprosy in its non-symptomatic, subclinical phase. However, molecular techniques such as PCR, are still not fully accepted; the interpretation of results are not conclusive, and PCR is expensive and not readily available in developing countries where the transmission of leprosy is a problem (Job et al. 1997, Torres et al. 2003). Detection of subclinical leprosy coupled with prompt and effective treatment could contribute significantly to global elimination of leprosy.

The detection of two new leprosy patients and infected HHC among high risk population, are evidence that the transmission of the leprosy infection still happens in a post-elimination region, despite the adoption of MDT during the course of 20 years. The classification of HHC according to IgM anti PGL-I titers and Mitsuda reaction should be useful to detect contacts at-risk to develop leprosy that can then be subjected to periodic follow-up; this fact should contribute to the global elimination of leprosy.

The objective of eliminating and ultimately eradicating the disease requires early detection and start of treatment. Failure to encourage early detection may extend the period in which an affected person remains infectious. It also increases the impact of the disease on the individual, with the consequent need for a greater duration of treatment and an increased risk of nerve involvement and potential disabilities.

\section{ACKNOWLEDGMENTS}

To Dr Berta N Restrepo-Jaramillo for her help in data analysis and Dr Piedad Agudelo-Florez for her comments and opinions.

\section{REFERENCES}

Chanteau S, Glaziou P, Plichart C, Luquiaud P, Plichart R, Faucher JF, Cartel JL 1993. Low predictive value of PGLI serology for the early diagnosis of leprosy in family contacts: results of a 10-year prospective field study in French Polynesia. Int J Lepr Other Mycobact Dis 61: 533-541.

Cho SN, Yanagihara DL, Hunter SW, Gelber RH, Brennan PJ 1983. Serological specificity of phenolic glycolipid I from Mycobacterium leprae and use in serodiagnosis of leprosy. Infect Immun 41: 1077-1083.

Feenstra P 2003. "Elimination" of leprosy and the need to sustain leprosy services, expectations, predictions and reality. Int J Lepr Other Mycobact Dis 71: 248-256.

Isenberg H 1992. Acid fast stainings. In Clinical Microbiology Procedures Handbook, 2nd ed., American Society for Microbiology, p. 7.2.1-7.2.4

Job CK, Jayakumar J, Williams DL, Gillis TP 1997. Role of polymerase chain reaction in the diagnosis of early leprosy. Int J Lepr 65: 461-464. 
Kalk A, Fleisher K 2004. The decentralization of the health system in Colombia and Brazil and its impact on leprosy control. Lepr Rev 75: 67-68.

Lockwood DN 2002. Leprosy elimination-a virtual phenomenon or a reality? BMJ 324:1 516-1518.

Meeker HC, Levis WR, Sersen E, Schuller-Levis G, Brennan PJ, Buchanan TM 1986. ELISA detection of IgM antibodies against phenolic glycolipid-I in the management of leprosy: a comparison between laboratories. Int J Lepr Other Mycobact Dis 54: 530-539.

Mora N, Pérez M, Beroliche M 1992. Determinación de anticuerpos anti-glicolípido fenólico I en población general de una área endémica de lepra. Fontilles de Leprología 6: 587-589.

Naafs B 2000. Viewpoint: leprosy after the year 2000. Trop Med Int Health 5:400-403.

Naafs B, Silva E, Vilani-Moreno F, Marcos EC, Nogueira ME, Opromolla DV 2001. Factors influencing the development of leprosy: an overview. Int J Lepr Other Mycobact Dis 69: 26-33.

Ottenhoff T 1994. Immunology of leprosy: lessons from and for leprosy. Int J Lepr 62: 108-121.

Pinto R 2000. El Control de la Lepra en Colombia. Informe Quincenal Epidemiológico Nacional, Ministerio de Salud, Instituto Nacional de Salud, Colombia.

Reich CV 1987. Leprosy: cause, transmission, and a new theory of pathogenesis. Rev Infect Dis 9: 590-594.

Ridley RS, Jopling WH 1966. Classification of leprosy according to immunity - A five group system. Int J Lepr 34: 225273.
Sengupta U 2000. Experience and lessons from the use of lepromin and Mycobacterium leprae-specific serology. Lepr Rev 71 (Suppl). S63-66.

Torres P, Camarena JJ, Gomez JR, Nogueira JM, Gimeno V, Navarro JC, Olmos A 2003. Comparison of PCR mediated amplification of DNA and the classical methods for detection of Mycobacterium leprae in different types of clinical samples in leprosy patients and contacts. Lepr Rev 74:1830 .

Van Beers SM, Izumi S, Madjid B, Maeda Y, Day R, Klatser PR 1994. An epidemiological study of leprosy infection by serology and polymerase chain reaction. Int J Lepr Other Mycobact Dis 62: 1-9.

Visschedijk J, van de Broek J, Eggens H, Lever P, van Beers S, Klatser P 2000. Mycobacterium leprae-millennium resistant! Leprosy control on the threshold of a new era. Trop Med Int Health 5: 388-399.

WHO 1998. Report of a meeting on the future role of leprosy training and/or research institutions, WHO, Addis Adaba, 25-26 Febraury.

WHO 2003. The Final Push Strategy to Eliminate Leprosy as a Public Health Problem. Questions and Answers, 2nd., Geneve

WHO 2004. Leprosy. Leprosy elimination project. Status report 2003, Geneva, p. 1-39.

Wu Q, Li X, Yin Y, Shu H, Wei W, Liu Q, Ye G, 1999. A study on the methods for early serological diagnosis of leprosy and their potential use. Int J Lepr Other Mycobact Dis 67: 302-305. 